\title{
Associative Multiple-Label Image Analysis Method for Synapse Identification in Neuronal Cultures: Application to Comparative Analysis of Synapse Formation Efficiency \& Distribution on Smooth and Topographically Modified Surfaces
}

\author{
H. Narasimha-Iyer*, N.M. Dowell-Mesfin**, M.-A. Abdul-Karim*, B. Roysam*, J. Turner** \& W. Shain** \\ * Rensselaer Polytechnic Institute, Troy, New York 12180-3590, USA. \\ ** The Wadsworth Center, NYS Department of Health, Albany, New York 12201-0509, USA.
}

The study of neuronal communication requires reliable detection and quantification of synapses. Neurons cultured on topographically modified surfaces that direct process growth provide a controlled observation mechanism [1]. In the absence of a direct fluorescent marker, synapses must be inferred from pre- and post-synaptic markers [2]. We have developed an automated quantitative method for identification of synapses using multiple fluorescent labels and associative image analysis. This method has been applied to comparative study of (i) synapse formation efficiency on smooth and modified surfaces; and (ii) spatial distribution of synapses relative to neuronal processes.

Hippocampal neurons isolated from embryonic rats were seeded onto smooth and colonnadepatterned polystyrene surfaces. Cells were fixed at 14 days in vitro and processed for immunocytochemistry. Antibodies were used to localize synaptophysin, a pre-synaptic protein (green channel in Fig. 1), and $\beta_{\text {III }}$ tubulin, present in the cell body and all neuronal processes (blue channel in Fig. 1). Flouresceinated phalloidin was used to detect F-actin (red channel in Fig.1), localized in highest concentrations in dendritic spines (where many synapses occur), and in growth cones. Three-color $z$-stacks were collected $(1040 \times 1392 \times 35 \times 3)$ on a wide-field epiflourescence inverted microscope with a $60 \times$ oil-immersion objective (1.4 NA) and $\mathrm{x}, \mathrm{y}$ and $\mathrm{z}$ resolutions of 0.1068, 0.1068 and $0.4 \mu \mathrm{m}$ respectively, and deconvolved (Autoquant, Inc., Watervliet, NY).

The maximum intensity projections from each channel were processed. The inter-channel bleedthrough was negligible, allowing separate segmentation of each fluorescence channel. The puncta in the synaptophysin and actin channels were segmented using an adaptive filtering algorithm, and connected components analysis was performed. Morphometric measurements were then extracted from the segmented regions. Growth cones were identified from the actin channel as regions with tubularity (ratio of major axis to minor axis length), area and mean intensity greater than $5,0.57 \mu \mathrm{m}^{2}$ (50 pixels) and 30 grayscale levels respectively, and were excluded from further processing. The neurites in the $\beta_{\text {III }}$ tubulin channel were traced automatically using a previously published algorithm $[1,3]$. Fig. 2 is an enlarged view of the segmentation results for the boxed region in Fig. 1.

Synapses were then identified by association of opposed pairs of puncta of the pre- and postsynaptic proteins. From the normalized frequency plot (Fig. 3), the distance between the synaptophysin and actin puncta for a synapse was estimated to be less than $0.75 \mu \mathrm{m}$ ( 7 pixels). This choice was validated visually by an expert observer. The Synapse Formation Efficiency (SFE) was computed as the ratio of the number of synaptophysin puncta that form a synapse to the total number of synaptophysin puncta (Fig. 4). The SFE was found to be $8 \%$ higher for the cultures on modified surfaces; suggesting that directed process growth may increase synapse formation. The mean distance from the centroids of the identified synapses to the nearest processes was observed to decrease by $7 \%$ for the modified surfaces. 


\section{References:}

[1] Dowell- Mesfin et al., J. Neural Eng. 1 (2004), 78.

[2] Obermair et al., Euro J. Neuroscience, 17 (2003), 721.

[3] Al-Kofahi et al., IEEE-TITB 7(4) (2003), 302.

[4] NSF grant EEC-9986821, NSF STC ECS-9876771, NIBIB R01-EB000359, \& NINDS R01-NS1044287.
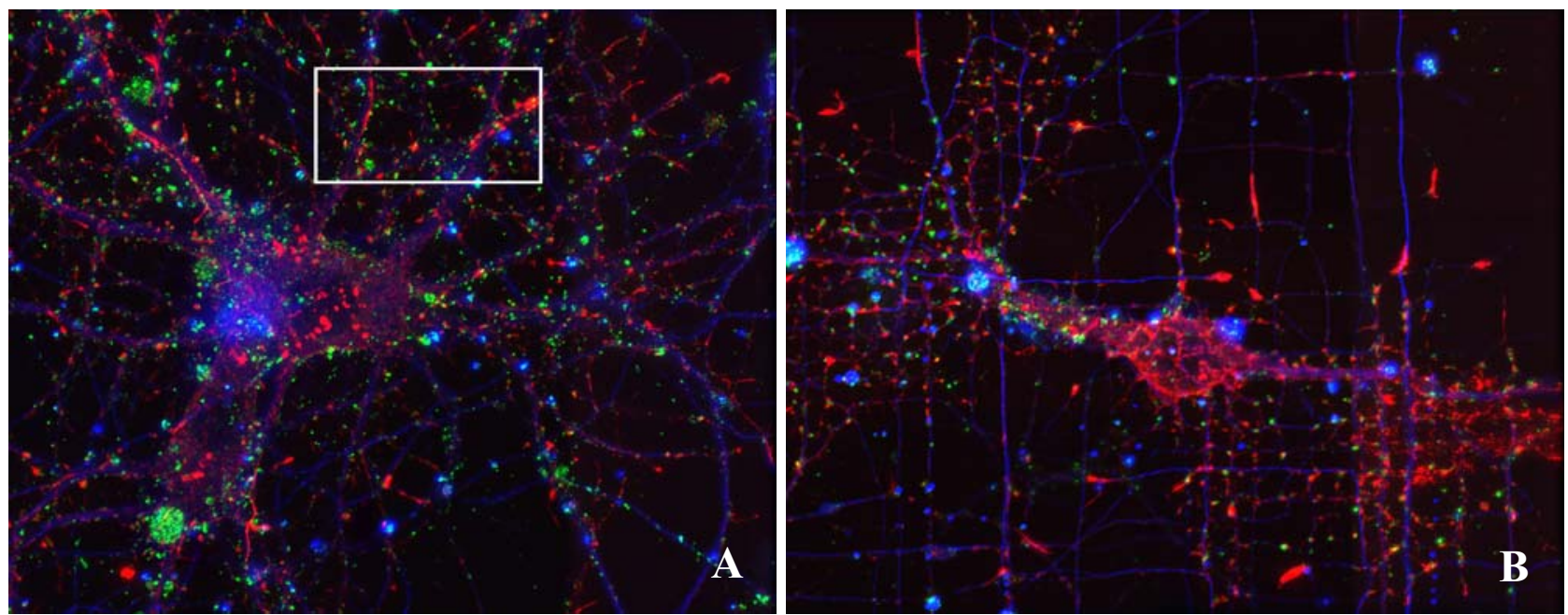

Fig. 1: Deconvolved \& corrected triple-label images of neurons cultured on: (A) smooth surface; (B) topographically modified surface. Red: Actin; Green: Synaptophysin; and Blue: $\beta_{\text {III }}$ tubulin.
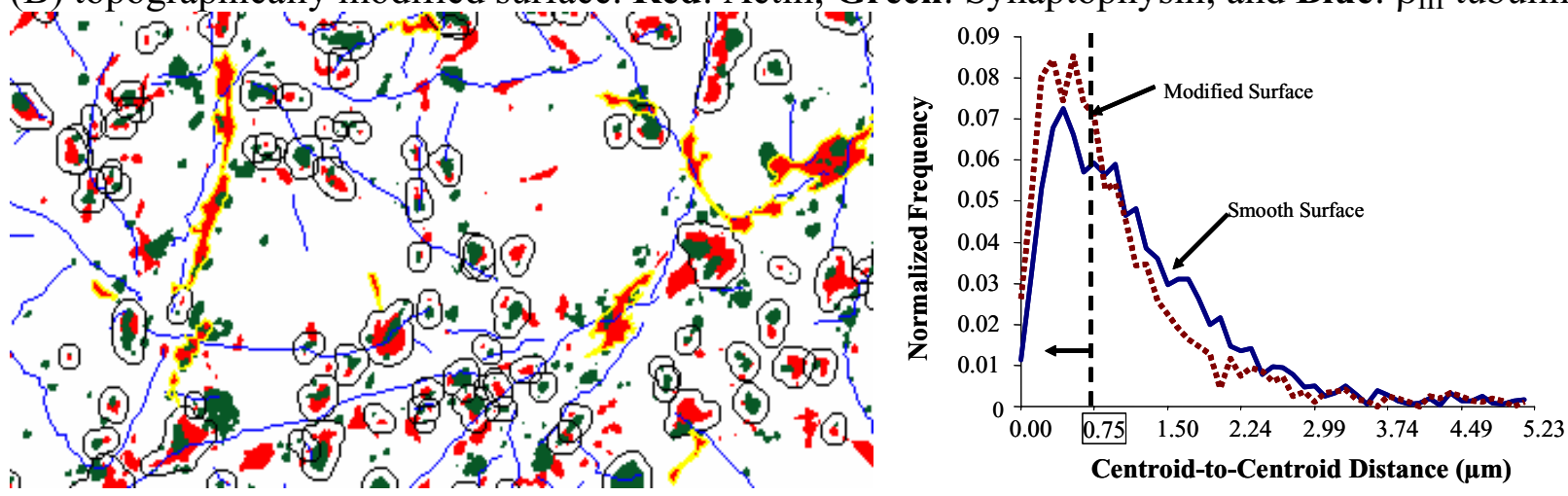

Fig. 2: Close-up view of segmentation of boxed Fig. 3: Normalized Histogram of the region in Fig. 1(A). Green: Synaptophysin; Red: distance between synaptophysin and actin Actin; Black outlines: Identified Synapses; Yellow puncta. Pairs less than $0.75 \mu \mathrm{m}$ apart are outlines: Identified growth cones.

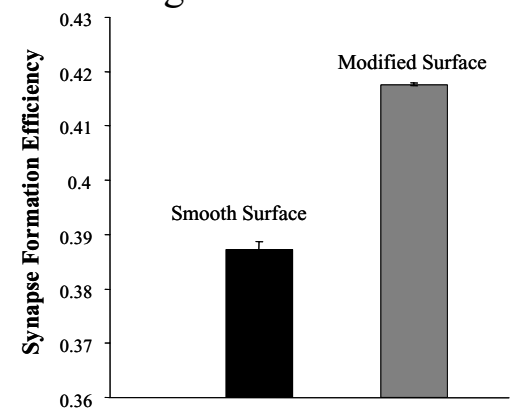
deemed to indicate a synapse.

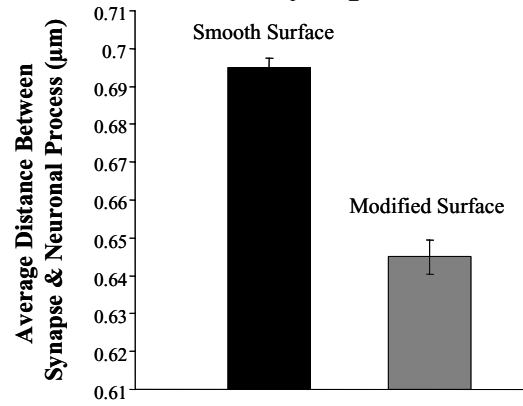

Fig. 4: Neuronal cultures on modified surfaces had 7\% higher synapse formation efficiency (left) and $7 \%$ lower average distances of the synapses to the nearest processes (right). 